\title{
Design of Robot-Inclusive Vertical Green Landscape
}

\author{
Matthew S. K. Yeo*(D), S. M. Bhagya P. Samarakoon (D), Qi Boon Ng (D) M. A. Viraj J. Muthugala (D) \\ and Mohan Rajesh Elara $\mathbb{D}$
}

\begin{abstract}
Engineering Product Development Pillar, Singapore University of Technology and Design, 8 Somapah Rd., Singapore 487372, Singapore; bhagya_samarakoon@mymail.sutd.edu.sg (S.M.B.P.S.); amanda_ng@sutd.edu.sg (Q.B.N.); viraj_jagathpriya@sutd.edu.sg (M.A.V.J.M.); rajeshelara@sutd.edu.sg (M.R.E.) * Correspondence: matthew_yeo@mymail.sutd.edu.sg; Tel.: +65-9070-0760
\end{abstract}

check for updates

Citation: Yeo, M.S.K.; Samarakoon, S.M.B.P.; Ng, Q.B.; Muthugala, M.A.V.J.; Elara, M.R. Design of Robot-Inclusive Vertical Green Landscape. Buildings 2021, 11, 203. https://doi.org/ 10.3390/buildings11050203

Academic Editor: Łukasz Sadowski

Received: 1 April 2021

Accepted: 30 April 2021

Published: 13 May 2021

Publisher's Note: MDPI stays neutral with regard to jurisdictional claims in published maps and institutional affiliations.

Copyright: (c) 2021 by the authors. Licensee MDPI, Basel, Switzerland. This article is an open access article distributed under the terms and conditions of the Creative Commons Attribution (CC BY) license (https:// creativecommons.org/licenses/by/ $4.0 /)$.
Abstract: Vertical gardens have emerged alongside the increase in urban density and land scarcity to reintegrate greenery in the built environment. Existing maintenance for vertical gardens is labour-intensive, time-consuming and is being increasingly complemented by robotic applications. While research has been focused on enhancing robot design to improve productivity, minimal effort has been done on 'design for robots' in creating suitable environments for optimal robot deployments. This paper proposed a multi-disciplinary approach that brings together architects, designers, and roboticians to adapt the design of the vertical garden infrastructure to counteract the limitations of the maintenance robot. A case study on an existing plant maintenance robot 'Urodela' was conducted to determine the limitations encountered by robotic aid during operation. A robotinclusive modular design for vertical gardens is proposed based on robot-inclusive principles, namely manipulability and safety, along with architectural design considerations. Design explorations for different configurations of track layouts of the proposed robot-inclusive modular design for vertical gardens is further analysed to validate its applicability and scalability.

Keywords: vertical green landscape; design for robot; robot-inclusive; vertical garden; manipulability; green wall

\section{Introduction}

Vertical green landscapes is a method for reinstating greenery into rapidly growing urbanised areas [1,2]. One of many effective ways to achieve more sustainable forms of development in our cities is through an increase in the diffusion of vertical green landscape technologies on roofs and walls of residential, institutional, industrial and commercial buildings. The implementation of vertical green landscapes and gardens offers numerous economic, social and environmental benefits such as air and water quality improvements, stormwater retention, operational cost savings, sound absorption, recreational opportunities and design aesthetics $[3,4]$.

Vertical gardens, a type of vertical green landscape, is defined as a system which plants are grown on vertical surfaces such as exterior and interior walls of buildings in an organised manner with systematic maintenance [5]. In the same way vertical gardens provide benefits on the urban scale, they also enhance energy efficiency, indoor environment quality, improve ambient cooling within the building. The vertical garden system differs from the green facade in that they receive water and nutrients from within the vertical support structure instead of directly from the ground. As vertical gardens comprise of more than one distinct type of plants, the transformation and adaptation of the vertical surface to support greenery is of particular interest to engineers, architects, planners and ecologists [6].

The maintenance of vertical gardens, however, is a strenuous activity which usually requires more care and maintenance compared to other types of vertical green landscapes. Maintenance tasks such as trimming and cutting of wild plant growths, plant replacement, 
pest control and watering is heavily reliant on human labour, and is often tedious and usually involves working from tall heights [7]. In recent years, substantial research development in robotics has been seen to play an increasingly important role in improving the quality of building maintenance, such as wall cleaning [8], facade cleaning [9] and floor cleaning [10]. Many developments of ground robotic and automation solutions for agriculture and gardening have proven the potential of improving agriculture through implementing robotics $[11,12]$. In the context of vertical garden maintenance, the applications of robotics could further reduce the strain on human labour and occupational hazards. A robotic arm intended for crop harvesting of vertical farms is proposed in [13]. Kim et al. [14] proposed an intelligent robot management system for vertical farms. The proposed robot management system can be used to establish the coordination between robots and a sensor network placed on a vertical farm. The cited robotic applications, though implemented for vertical gardens, do not possess the ability to access its entire vertical workspace. On the other hand, while accessibility to the workspace can be reinforced with the deployment of unmanned aerial vehicles, this technology is currently unable to perform maintenance with its low payload capabilities [15].

State-of-the-art approaches such as the Wallbot [16] and Urodela [17] discuss the potential of robots with wall-climbing capabilities in vertical garden applications due to their ability of reaching extended heights. The Wallbot adheres itself onto the vertical landscape using cables hung in cross-wise fashion to the vertical plane to inspect plants. Urodela [17] is a robot operated on a rail track fixed on a vertical gardens. The rail structure allows the movement of the robot to cover a wide area on the vertical plane. Even though these climbing robots can cope with extended heights, these robots still face issues that degrade their productivity in most of the existing vertical garden designs. For example, the motion and movement ranges are still limited by their cable lengths or rail track designs.

Robotic applications in any form of maintenance, requires the integration of both the robotic functionality and its working environment, or in this case, for vertical gardens. Robotic technology can be sub-categorised into its system components or functionality such as manipulator mechanism [18], control [8], sensing techniques [19] and mobility [20]. In many cases, the environment in which the robot operates in dictates the way such system components are designed. However, the environmental conditions can largely vary, which may result in the reduction of productivity in its deployment. Conventionally, buildings are designed with consideration of human factors and ergonomic principles to improve the productivity of work done by its occupants [21,22]. Based on these inspirations, the works $[23,24]$ examined the possibility of improving the productivity of robots through implementing robot-inclusive architectural design changes instead of solely improving the functionalities of the robots These cited works suggested that the robot-inclusive architectural design could facilitate performance improvements of robots. This design process of robot-inclusive building infrastructure is normally referred as 'Design for Robots' [23,25]. Designing for robots allows the implementation of robotics technology in more areas of work to complement human labour and reduce safety hazards, eventually boosting productivity and outputs. This is mainly achieved by modifying spatial layouts, choosing and integrating robot-inclusive designs into the building architecture. However, little or no work has been done on the design of spatial and infrastructural aspects to allow for robot-aided maintenance and inspection in vertical gardens.

This paper seeks to fill the gap in robot-aided maintenance infrastructure design. A novel design framework for robot-inclusive vertical gardens is developed and derived from a study of the limitations in maintenance robots operating in such environments. Specifically, a case study is conducted based on the Urodela robot [17] to understand in detail its components and spatial limitations that hinders its productivity. Following this, the limitations are factored into design principles mainly manipulability and safety [23]. This paper also seeks to apply the design guidelines in an exemplary vertical garden in the context of the Urodela robot. The approach seeks to bridge the knowledge across 
roboticians, architects and designers to consider robot-aided vertical garden maintenance and inspection in their design.

The paper is organised as follows: A summary of case studies on existing vertical green landscaping is given in Section 2. Robot-inclusive design principles relevant to this design is outlined in Section 3. Section 4 discusses the Urodela vertical landscape robot as a case study to understand current limitations with the green wall robotic maintenance process. Section 5 generates the design considerations for robot-inclusive vertical garden design from both the robotic and architectural aspects, and explores the proposed design for modular vertical green landscape. Section 6 then discusses about the validation and analysis of the proposed design. Lastly, Section 7 concludes the paper with future directions.

\section{Vertical Green Landscape Maintenance}

Vertical green landscapes can be classified into two categories: green facades or vertical gardens. The main difference between green facades and vertical gardens is the location of its source of plant growth. Green facades rely on plants rooted in natural ground, and are typically known as ground-based greening methods whereas vertical gardens are known as wall-based greening methods where plants are directly planted on the wall [26]. Without the requirement of the rooting space at ground level, vertical gardens provide much more flexibility in terms of vegetation types and are able to create more controlled plant growth environments for systematic maintenance [1]. There are three different types of vertical gardens: continuous, modular and linear systems. Continuous vertical gardens depend on a single support structure while modular gardens depend of several modular elements stitched together to form the entire vertical landscape. Linear vertical gardens rests on cascading elements linearly affixed to the wall.

In terms of design, vertical gardens are mainly composed of mechanical and structural elements comprising of structural support frames, growth media by way of fabrics or substrate layers, waterproof membrane and an irrigation and drainage system [26]. Irrigation networks for vertical gardens are usually more complex as compared to green facades. With the placement of various plants across the green landscape, the water requirements for the various species vary widely and thus involves several irrigation lines at different heights [27]. However, the main obstacle lies in the maintenance process as operations like fertilising, pruning, pest control and disease management has to be performed at tall heights. The Bosco Verticale in Milan, an example of a linear vertical garden. The residential complex consists of two towers, sprawled with more than thirteen thousand plants, on both towers' facades [28]. The towers are distinguished by multiple overhanging and alternating balconies to situate various species of plants and trees. Vertical garden maintenance on the Bosco Verticale is heavily reliant on skilled human workers. At a height of over 80 metres, the towers require a specialised team of arborist-climbers to abseil down the building, carrying out yearly pruning and inspection [29]. This form of maintenance is not universally applicable and may also result in additional unfavourable operational costs.

With the rapid growth of green infrastructure in cities, robotic applications present itself as a proven solution to complement or reduce the need for human labour in terms of inspection and maintenance. Robots, designed with different modes of locomotion such as crawling, climbing or flying, are capable of performing tasks at locations difficult or dangerous to reach by humans. Advanced applications constructed with in-built sensors are able to perform operations autonomously, allowing for regular inspection and maintenance, thus minimising human intervention.

This paper focuses on the aspects of wall-climbing robots for vertical garden maintenance, specifically the Urodela robot [17]. The Urodela robot was designed to be integrated into an interior vertical garden that is to be showcased in the Dubai Expo Pavilion 2021. While the customisation of the robot would lead to optimal operation in its environment, such designs limit its applicability in other vertical garden typologies. Similarly, designers of vertical gardens focus on the optimisation of benefits that vertical gardens can provide for buildings. Momtaz [30] discusses design considerations in planning of vertical gardens 
such as aesthetic and urban design, vertical garden type, plant material selection, noise reduction, reduced energy use, temperature control and costs. As technology and architecture improves over time, design considerations for robot applications within building infrastructures like vertical gardens have to be addressed. This paper seeks to propose design guidelines for robot-inclusive vertical gardens that reduces the complexity of robot design as well as maximise its operational productivity.

\section{Robot-Inclusive Design Principles for Architecture}

Robot-inclusive design considers both robot and human ergonomics to determine ways to improve the design of surrounding objects and the areas of the robot's workspace to improve the robot's performance. This approach brings together roboticians and architects to integrate the behaviour derived from the structure of spaces with that of the robot. Based on challenges posed by the human environment, roboticians typically seek to overcome the current limitations of the robot by improving on its functionality such as robot perception [31], reconfigurable design [32], human-robot interaction [33], control [34], and energy efficiency [35]. Instead of increasing the complexity of the robot design based on customised solutions for the robot, those challenges could be easily addressed following a top-down approach that changes the spatial environment in which the robot operates in instead, to better fit the environment to the robot's tasks instead of solely upgrading the robot without considering its working area.

The paper derives robot-inclusive design guidelines from the five design principles that were inspired by universal design methods [36,37], namely: accessibility, safety, activity, observability, and manipulability. Accessibility deals with the aspects of the area covered by the robot, to improve barrier-free access and connection for robots to travel for their tasks. Safety guidelines for robot-inclusive environments involves reducing damage to the robots during its operation. Activity refers to the provision of the simultaneous traffic flow for people, goods and robots. Observability involves the improvements made in the spatial environment for robot visibility and perception of its surroundings for navigation. Manipulability comprises of the changes made to enhance the robot's ability to move or rearrange objects in the robot's given environment, if required. The five design principles are informed by spatial constraints observed from robot deployment and design. Based on the study of the vertical landscape design, design parameters for robots such as fixtures, qualities in the space for the robots' sensors to detect, spatial geometry and wall surfaces are assessed.

\section{Case Study of Vertical Landscape Robot: Urodela}

Our case study involves a vertical garden landscape that acts as a living green wall typically situated in commercial and retail buildings. The study aims to incorporate the Urodela robot for plant maintenance such as pruning, identification and replacement of defective plant pots. Based on the understanding of the Urodela robot and spatial characteristics of the vertical landscape, design guidelines categorised under the five principles are inductively derived. Design guidelines under manipulability and observability considers the vertical landscape to allow the robot to visually and physically access the largest possible area of the planted zones on the vertical landscape in order to maximise the maintenance capabilities for the robot. The colour of the rail track can be adjusted to fit the overall colour scheme of the building to allow the Urodela robot to blend seamlessly with the vertical landscape, in accordance to the robot-inclusive principle for activity. Another important design guideline for robot-inclusivity is to provide additional safety measures such as support structures for the robot to prevent it from falling off the track, or improve the manipulator efficiency by modifying the structures it interacts with, so as to make it more efficient for the robot to complete its tasks. Under accessibility, design guidelines consider the ability of the rail track to support the weight of the robot as it traverses over the vertical landscape. 
The Urodela robot [17] is considered for this paper as part of a case study for the proposed robot-inclusive design for vertical green landscapes. The design of the robot is shown in Figure 1. Mounted on a fixed dual round-profile track on the vertical landscape, it is designed to traverse over the vertical green landscape to perform manipulation tasks such as pruning and plant pot exchange with its manipulator arm. Urodela consists of three bi-wheeled modules connected on a flexible common axis, which provides the robot with its structure, adhesion and its ability to traverse planar bends on the rail tracks. In order to ensure sufficient grip and safety on the rail track structure, the locomotion system of the Urodela robot is made up of multiple sets of wheels mounted in multiple orientations that wrap around the track rails as it travels along a vertical surface, as seen in Figure 2. The robot's outer shell covers the interior modules during its operation, and also protects the components underneath it from weathering from outdoor usage. The cover would preferably be designed after the appearance of insects to fit the intended theme of insects climbing along a planted surface. It also acts as a storage cover to contain the collapsed arm manipulator when it is not in use.

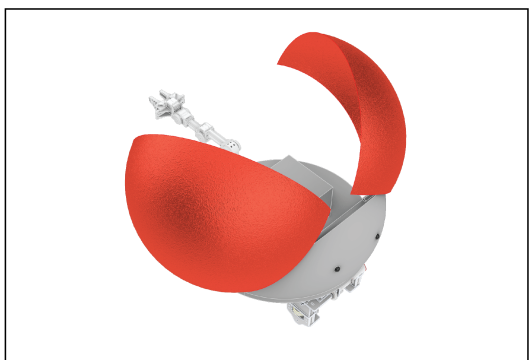

(a)

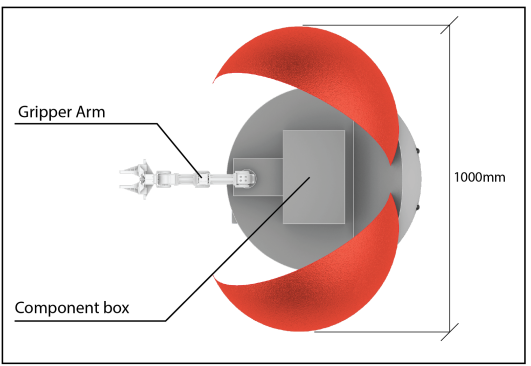

(c)

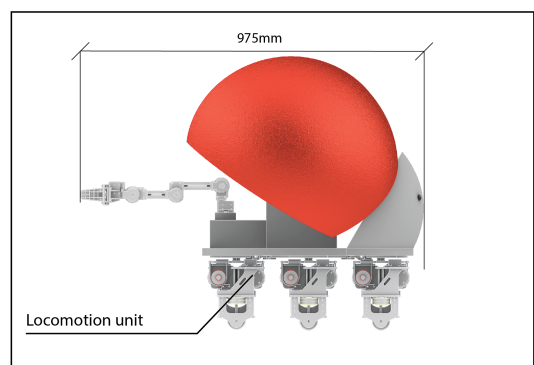

(b)

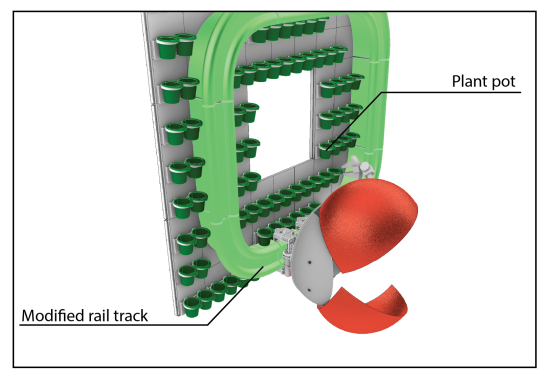

(d)

Figure 1. (a) Overall robot model view, (b) Side view, (c) Top view, (d) Robot on designed track.

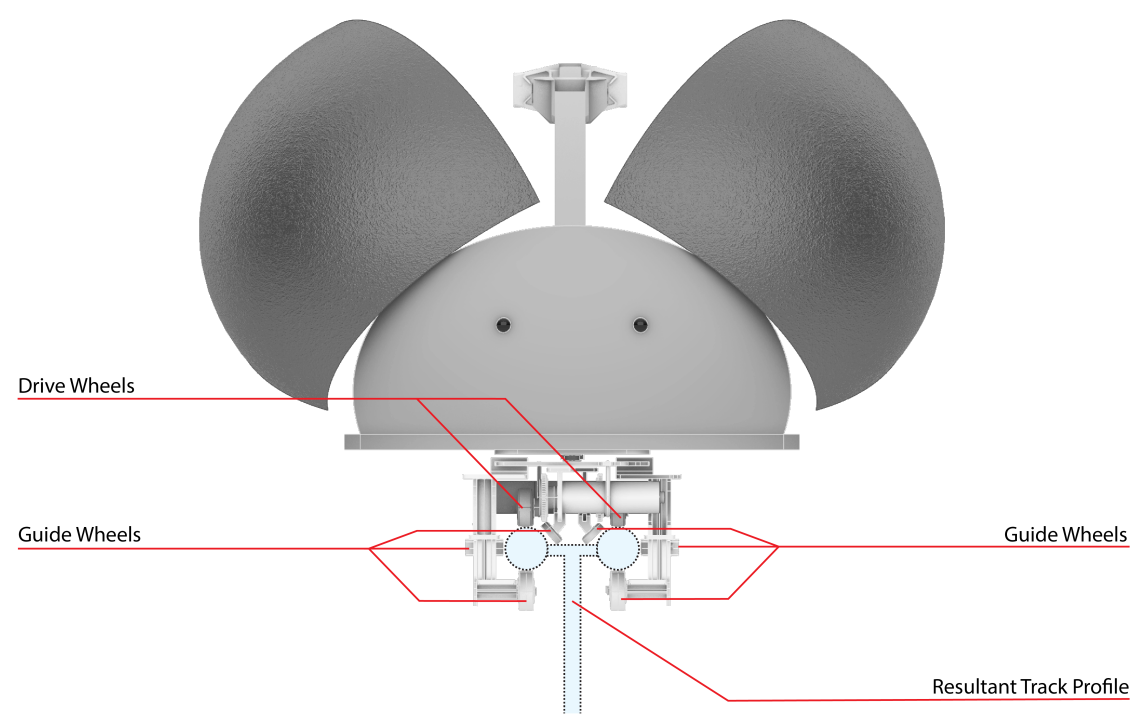

Figure 2. Front view of robot showing wheel mechanism. 
The main body is made of machined Aluminium-6061, and has wheels for locomotion, and magnets (Lifton magnets, NA32) for adhesion along the vertical surface. The magnets secure the robot to the ferromagnetic tracks which prevents it from falling off from the vertical plane due to gravity. The strength of magnet depends on the robot's overall weight and its distance from the ferromagnetic tracks that secures the robot to the track during its operation. The robot's mode of locomotion utilised customised rubber wheels with concave wheel profiles that allows them to maximise its contact area with the round track profile. Moreover, two free-rolling spring-loaded guide wheels on each side of the locomotion modules help the robot locomotion system to grip the sides of the rail tracks. The springloaded nature of the guide wheels allows the robot to circumvent uneven track surfaces if present. The robot travels on a dual round-profile track of $60.2 \mathrm{~mm}$ diameter raised above the vertical surface by T-profile struts, confining the current design of the rail profile and track apparatus to accommodate its wheeled system. According to the specifications of the robot, the robot's maximum turning angle is $20^{\circ}$ between adjacent modules. With the standard build of three bi-wheeled modules connected together, this effectively allows the robot to travel on arcs up to a maximum of $40^{\circ}$.

The Urodela robot has a mounted manipulator with five degrees of freedom and a two-finger gripper to better grasp and manipulate the plant pots with diseased or defective plants from various angles and heights within its range of operation. The gripper of the arm can be interchanged with other tools to help the robot perform other maintenance tasks, such as pruning, watering of plants, or scanning of plant health using a sensor array. The sensor arrays enables the robot to perform detection of disease or damage on the floras.

\section{Robot-Inclusive Vertical Gardens}

\subsection{Design Guidelines for Robot-Inclusive Vertical Gardens}

The paper proposes a robot-inclusive design that focuses on maximising the reachability and grip of the manipulator arm of the Urodela robot (manipulability principle) and improving its safety in operation. Manipulation tasks form the main function of the Urodela robot acting upon vertical landscapes, performing otherwise laborious tasks such as plant pot replacements at tall heights. According to the design of Urodela robot given in [17], certain factors of the robot's inherent properties inform design guidelines for a robot-inclusive vertical garden infrastructure.

\subsubsection{Improving Manipulability}

Autonomous skillful manipulation is essential for the manipulator arm of the Urodela robot to effectively prune, grip and replace pots. The design of the vertical garden infrastructure would have to take into account the effective working range of the manipulator arm. Considering this, a set of design guidelines to utilise the maximum effective working range of the manipulator arm of the Urodela robot are defined as follows:

1. Plant pots on the vertical garden should be installed at reachable and consistent heights and arranged within working range of the manipulator arm.

2. The form and shape of the plant pots should be easy to reach, grasp, move, arrange, operate, or control.

3. The mass and material of the plant pot should be lightweight to allow ease of pot replacement.

4. The rail track should be installed at a height suitable for easy reach to the plant pots.

In our case study, the Urodela robot utilises the Robotis OpenManipulator-X (RMX52-TNM) which has a maximum span of $380 \mathrm{~mm}$ and five degrees of freedom. It was determined that a track height of $10 \mathrm{~cm}$ as measured from the base of the vertical garden infrastructure was suitable to provide sufficient reachability for its arm manipulator to grasp the plant pot installation without expending excessive energy, whilst allowing for sufficient clearance for the robot's locomotion system to pass over on the rail track without colliding with any of the plants on the vertical garden. The variations to the height of the rail track are illustrated in Figure 3. 


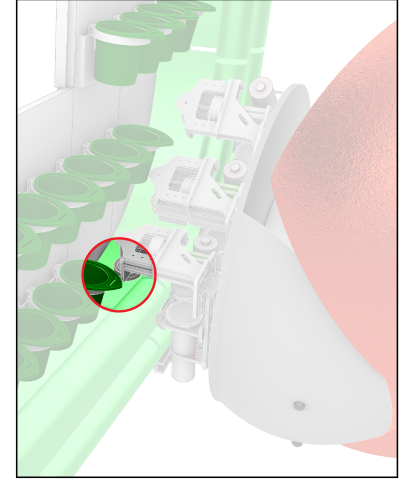

(a)

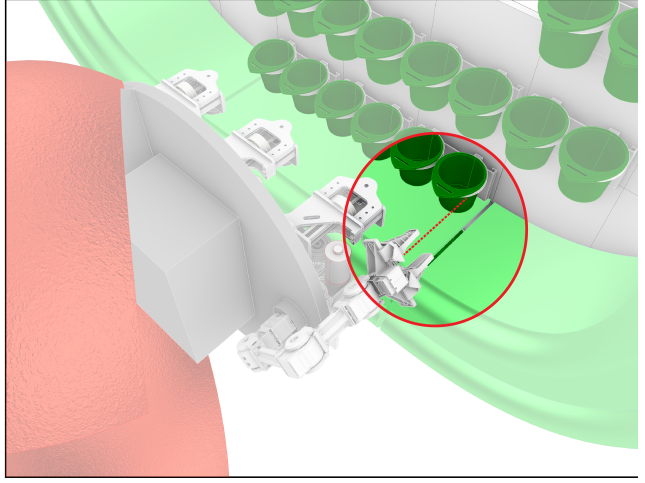

(b)

Figure 3. (a) Robot collision with plant pots if track height is too low, (b) Inability of robot to reach plant pot if track height is too high.

\subsubsection{Enhancing Safety}

By creating a safe environment for operation, the robot can then perform and complete its tasks without endangering the other parts of the its system, or its human operators. With these considerations, a set of design guidelines to improve safety of the Urodela robot during its operation are defined as follows:

1. The turning radius of the rail track cannot exceed the turning limits of the robot to prevent over-straining its locomotion components and allow smooth traversal.

2. Vertical orientation of the rail track layout for robot traversal on the vertical garden should be kept to a minimum to reduce likelihood of wheel slippage.

3. Turning points of the rail track should be kept to a minimal to prevent expending unnecessary energy.

4. The design of the rail track should include a terminal point for robot replacement and removal.

5. Sufficient protection from human pathways should be implemented to prevent human-robot collisions.

Based on the standard build of three bi-wheeled modules of the Urodela robot, the turning radius within the track design has to be kept to a limit of $70 \mathrm{~cm}$. Even though turning bends and vertical traversal in the track path are essential to improve area coverage of the vertical garden, it may not be ideal as it rapidly causes wear and tear on the wheels.

Terminal points on the rail track should be provided for the Urodela robot to dock, secured with additional supports to prevent the robot from slipping. They also provide ease of access to detach and replace the robot for future deployments.

\subsection{Proposed Modular Robot-Inclusive Vertical Garden Design}

This paper seeks to apply the design guidelines illustrated in Section 5.1 on an exemplary vertical garden in which the Urodela robot operates in. In this proposal, a novel modular track tile system is adapted to the Urodela robot to enable scalability and customisability of vertical garden design. The modular system is made up of multiple planar tiles which includes either straight or curved track sections, with an array of plant pots. The tiles can be linked together to create a continuous track path for the Urodela robot to traverse upon and perform maintenance tasks on the plant pots mounted within the tiles. With the design of the modular track tile system, architects and designers would be able to create different layouts of the rail track to fit on multiple forms of planar wall profiles. Based on the manipulability and safety guidelines as mentioned in Section 5.1, the design of the rail track, plant pot and the arrangement of the plant pots are defined in the following sections below. 


\subsubsection{Design of Rail Track}

Rail tracks that allow for robot movement is attached on each modular tile by means of welded T-shaped profiles at its endpoints and spaced intervals to reduce the overall weight of the mounted track, while still allowing for sufficient connection points to the tile. From the specifications set out in [17], the mounting brackets and rail track are made of steel alloy AISI 1020. The tracks are offset slightly from the tiles' edge to allow for thermal expansion of the metal track, especially when the setup is exposed to outdoor heat from the sun, whilst still allowing the robot to cross adjacent tiles without slippage or being stuck. As mentioned in the earlier section along with the specifications from the Urodela robot, the height of the track has been defined as $10 \mathrm{~cm}$ and the turning radius limit is set to $70 \mathrm{~cm}$.

Track terminal points are added to the track design to provide additional safety measures to keep the robot stationary and prevent it from falling off upon docking. Support structures and a hinged end track cap as seen in Figure 4 are installed at the terminal points for convenient detachment or replacement of the robot after the maintenance tasks have been completed. A modular tile for the terminal point of the track is defined for a more holistic approach to the customisability of the overall vertical garden design. The tile consists of an aluminium base, a truncated track, two support struts and an end track cap. The end track cap is held in place with a lockable clasp to either keep the robot at the end point of the track, or swivel outwards to allow the robot to be removed or turned around manually by the robot operator to reverse the robot's direction along the track, as seen in the diagrams (c) and (d) of Figure 4.

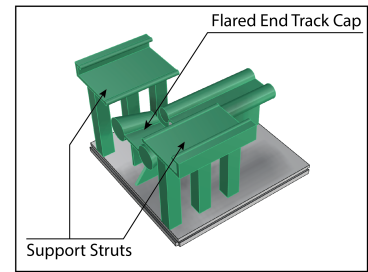

(a)

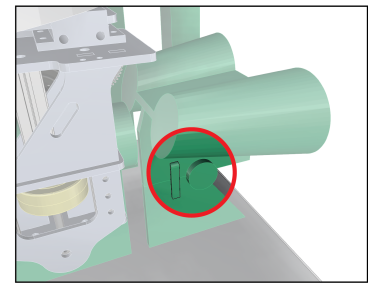

(d)

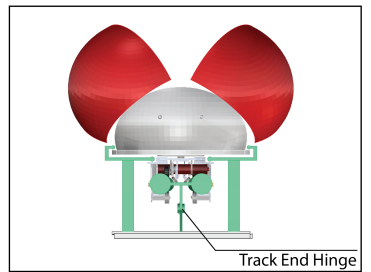

(b)

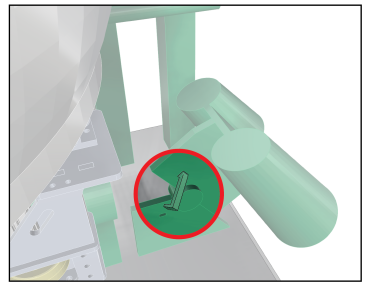

(e)

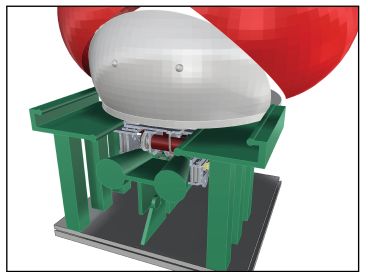

(c)

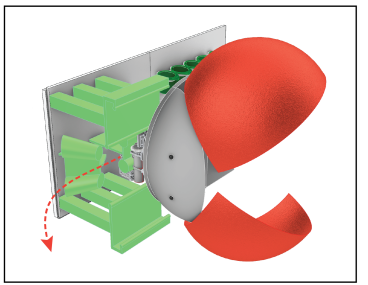

(f)

Figure 4. (a) Axonometric view of end tile, (b) Side view of support struts holding robot, (c) Close-up of robot stopped by flared track end cap, (d) Closed clasp during operation to prevent robot leaving track, (e) Open clasp for robot removal from track, (f) Method of removal for robot.

\subsubsection{Plant Pot Array Setup}

The design of the plant pot is adapted to cater to the $500 \mathrm{~g}$ payload of the manipulator arm and provide easy grip during maintenance. Figure 5 illustrates the plant pot component setup and assembly method. Clips are installed on plant pot holders to latch onto racks affixed to the base tiles. Each plant pot includes an extended lip to allow the arm manipulator to grasp onto when a plant pot for convenient replacement. Some plants suggested for their robustness in a tropical climate include, but are not limited to, are the ferns such as Polyscias fruticosadwarf (Parsley Panax), Selaginella kraussiana 'Brownii', Nephrolepis exaltata (Boston fern), Platycerium ridleyi (Ridley's Stag horn fern) and Asparagus densiflorus 'Sprengeri' (Sprengeri fern). Some examples of flowering plant species for the green wall would be the Aeschynanthus speciosus hook (Lipstick vine) and Allium fistulosum (Bunching Onion). Based on the soil volume requirements and the 
payload requirement of the manipulator arm, plant pots sizes are designed to fit small or medium-sized plants with a diameter of $8 \mathrm{~cm}$ and depth of $8.5 \mathrm{~cm}$. Lightweight material such as fiberglass or resin are also recommended to reduce the overall weight of each plant pot. With regards to the arrangement of the plant pots on each modular tile, the location and number of plant pots are dependent on whether the shape of the rail track structure.

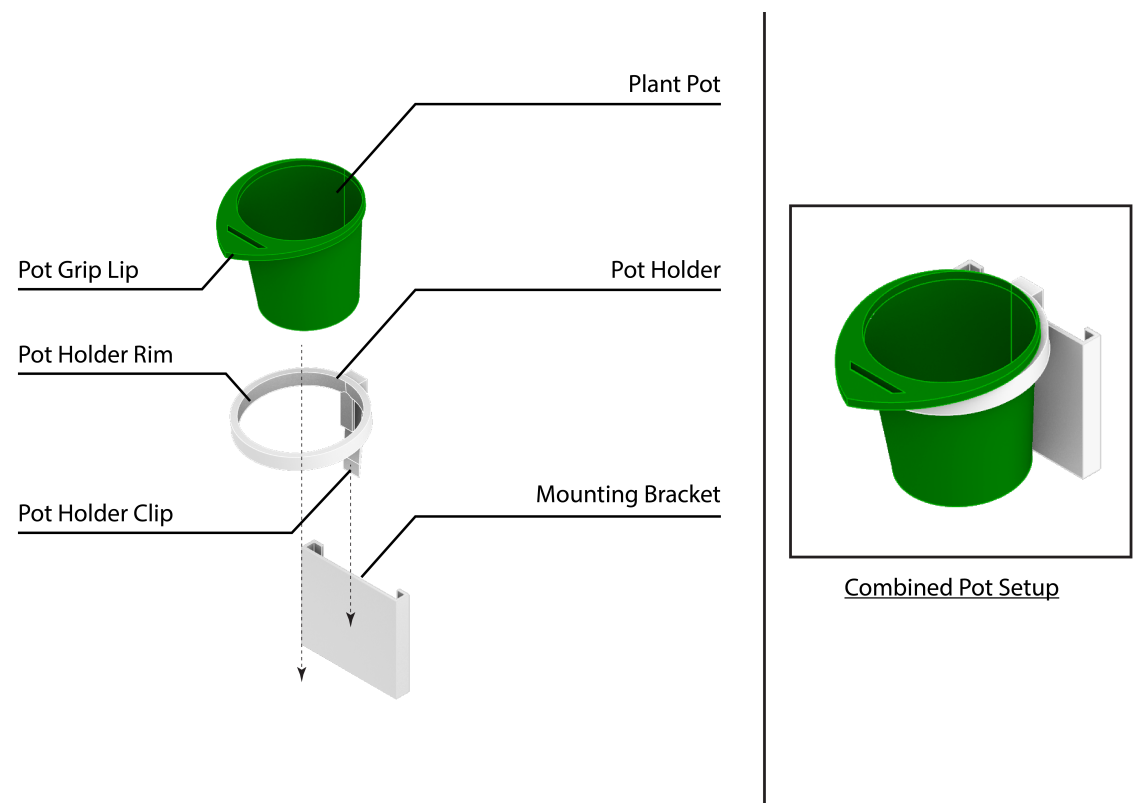

Figure 5. Assembly and parts of plant pot module.

5.2.3. Scalability and Customisation for Robot-Inclusive Modular Green Landscaping Tiles

The dimensions factored into each modular tile design is dependent on the rail track and the plant pot array setup. A total of eight unique tiles are designed for each type of planar directional change (four in total), straight track tiles (horizontal and vertical, two in total) and track ends in the horizontal direction (two in total) for a total of eight unique tile types. All tiles were determined a dimension of $60 \mathrm{~cm}$ by $60 \mathrm{~cm}$ as highlighted in Figure 6 . Tiles with track ends are designed to end only in the horizontal direction to minimise the chances of the robot falling off on a vertical track end with a spoilt clasp mechanism. Tiles with curved tracks allow for directional changes in the planned track layout, while tiles with straight tracks provide the reach across distances. Tiles with terminal track ends provide the locations whereby the robots can be replaced or removed.

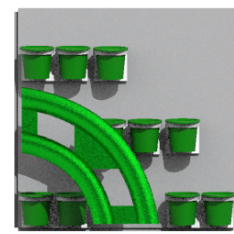

(a)

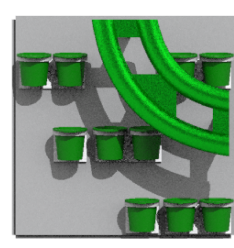

(b)

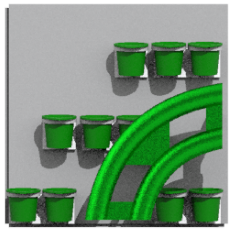

(c)

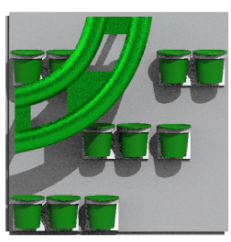

(d)

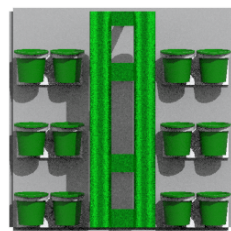

(e)

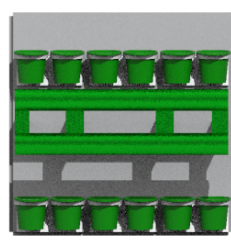

(f)

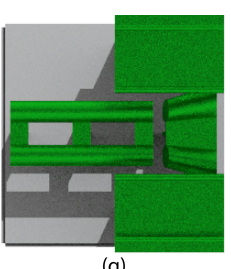

(g)

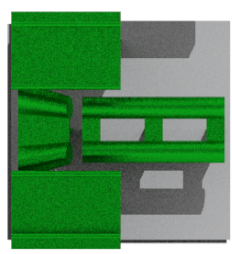

(h)

Figure 6. (a) SW bend tile, (b) NE bend tile, (c) SE bend tile, (d) NW bend tile, (e) vertical track, (f) horizontal track, (g) East track end, (h) West track end. 
Grooves and overhangs on the edges of each tile, seen in Figure 7, allow easy connections to each adjacent tile. This enables the contractors to have a systematic way to lay tiles during the installation process, as seen in Figure 8. Two consecutive curved-track modular tiles can be placed adjacent to each other in order for the robot to turn for $180^{\circ}$ in a U-bend, or four for a S-bend for the track along the wall. However, it would be recommended for designers to separate bends with straight track sections to reduce wear and tear on the robot's wheels from circumventing successive tight corners and bends in close proximity. This proposal also complements the modular vertical garden, particularly a grid panel system which can facilitate targeted replacements in case of damage. The irrigation system and addition of substrate for each plant pot will be facilitated by the Urodela robot, as opposed to the existing transport systems for modular tile green landscaping.

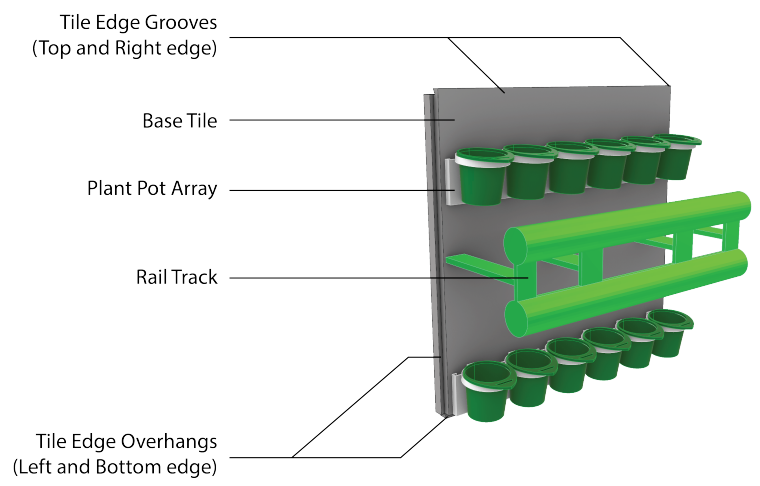

Figure 7. Groove and overhang system, closeup of a typical tile.

(a)

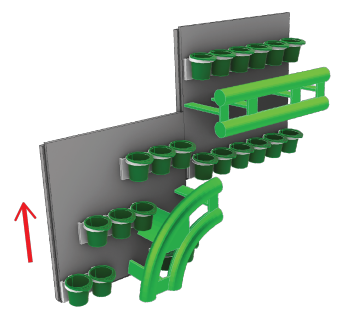

(b)

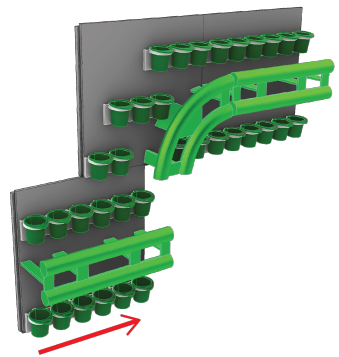

(c)

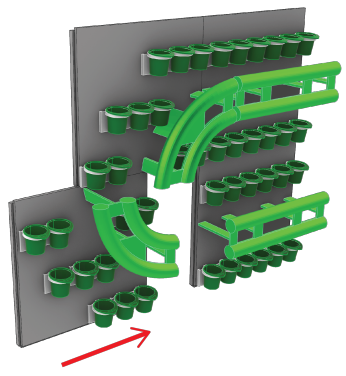

Figure 8. Installation process of modular green wall tiles, (a) Initial tile to be placed at top right corner of intended track surface, (b) Subsequent tiles are added from right to left, then from top to bottom due to design of overhangs and grooves, (c) Sequence of installation has to be done beforehand to ensure correct and continuous track path installation.

The proposed modular vertical garden design caters for robotic aid in the maintenance process. This concept seeks to reduce the reliance on human labour, operational costs and improve overall workplace safety. Furthermore, designers can easily rearrange the layouts of the modular tiles to form different track routes. The form-work for the modular tiles can be reused for multiple copies of the required tiles, as compared to that of a single-use non-modular track design, of which its form-work would be harder to dispose or reused for other purposes. This eventually results in less resources being used in the production and fabrication stage because of the modular nature of this rail track tile design. The simple replacement of damaged tiles, or reuse of tiles could be a form of sustainable practice. Vertical surface traversal also facilitates the ability for regular inspection and maintenance that can be done remotely with minimal direct human intervention. This state-of-the-art technological innovation could be applied to reduce barriers to adoption. 


\section{Results and Discussion}

\subsection{Validation of Performance Gain of Proposed Robot-Inclusive Vertical Landscape Design}

The main motivation of this paper is to propose a robot-inclusive green wall design, where the robot can perform better in terms of arm manipulability and safety. The reachability of the arm for plants is one of the critical factors which determines the efficiency of the robot carrying out its maintenance tasks. Therefore, the robot arm's reachability toward pots for the proposed modular tile design was examined. Ten different test layouts were created for assessing the robot's arm reach to pots. Five cases that follow the introduced design guidelines and five cases that do not follow the design guidelines were considered. The height of the track, pot arrangement, and track layout were varied for creating the test cases (for both positive and negative cases). Simulations were conducted using Matlab for evaluating the robot's arm reachability in the different configurations. In this regard, the kinematic model of the robot manipulator and movement restrictions imposed by the robot base were utilised for determining the effective workspace.

The set of test layouts created according to the guidelines of the introduced robotinclusive vertical garden design is given in Figure 9. The corresponding results of the robot reachability evaluation are given in Figure 10. The set of control layouts (i.e., layouts that are not designed per the introduced design guidelines) used for comparing the robot reachability is given in Figure 11. The corresponding robot reachability results are given in Figure 12. The robot reachability of each case is annotated in shaded color. All the test cases consisted of tiles of $60 \mathrm{~cm} \times 60 \mathrm{~cm}$ dimensions. A different number of tiles and combinations were integrated for making various layouts for the robot reachability evaluation. The track height of all the layouts was $10 \mathrm{~cm}$ except the layout design ' $b$ ' of the control test cases.

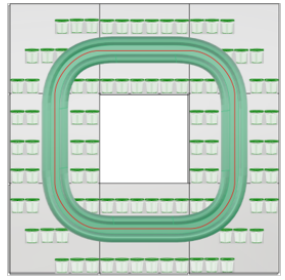

(a)

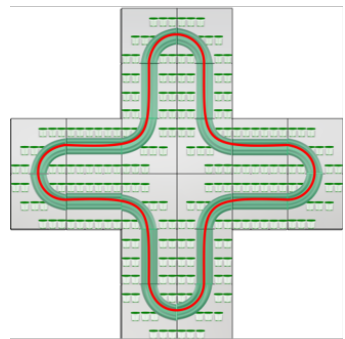

(c)

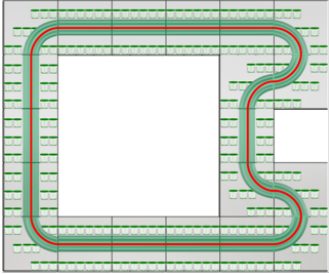

(b)

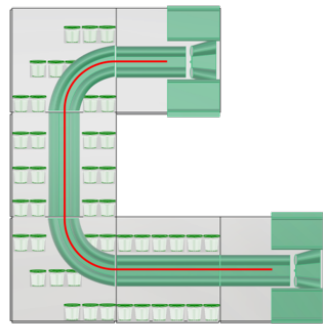

(d)

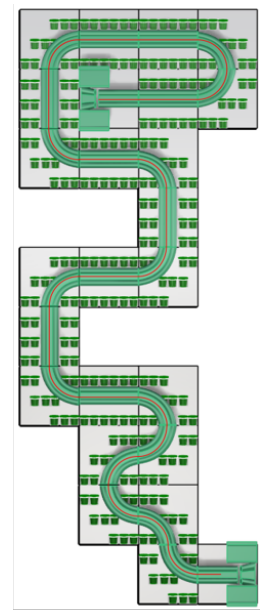

(e)

Figure 9. Vertical garden layouts designed considering the introduced guidelines. The track height is $10 \mathrm{~cm}$ for all the cases. $(\mathbf{a}-\mathbf{c})$ : closed track designs. (d,e): open track designs. It should be noted that the figures are not in the same scale.

The robot reachability analysis for test case 'a' of layouts created according to the guidelines is given in Figure 10a. According to the simulation outcomes of the reachability analysis, all the pots in the layout can be accessed by the robot (pot reachability is 100\%). Similar to case ' $a$ ', in the layouts ' $b$ ' and ' $c$ ' of the layouts (see Figure 10b,c) that followed the guidelines, which have closed complex track designs, the robot can reach all the pots using its manipulator arm. The cases ' $\mathrm{d}$ ' and ' $\mathrm{e}$ ' of the layouts that followed the guidelines (see Figure $10 \mathrm{~d}, \mathrm{e})$ represent track designs with track ends. As per the design guidelines, special 
attention has to be paid to the design of the track ends since the robot reachability is limited. Thus, these simulation results validate the ability to assure the robot reachability for all pots, even in complex closed or open track designs, if the introduced design guidelines are utilised for creating the layouts.

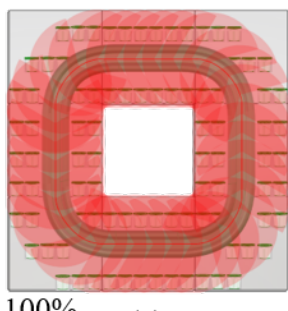

(a)

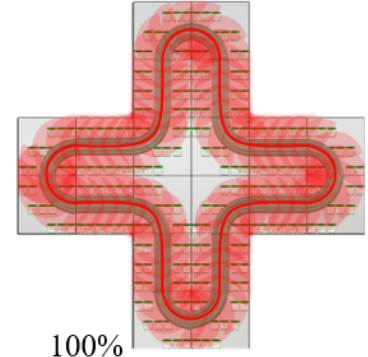

(c)

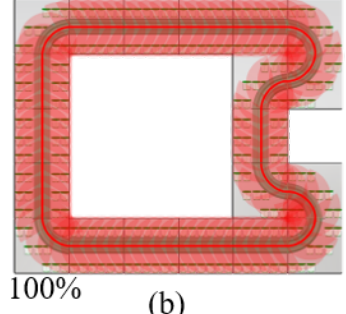

(b)

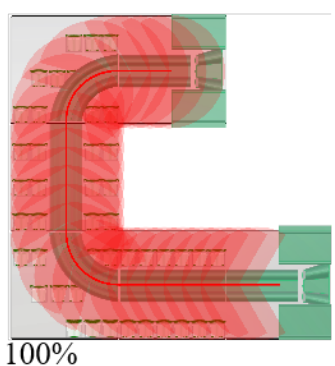

(d)

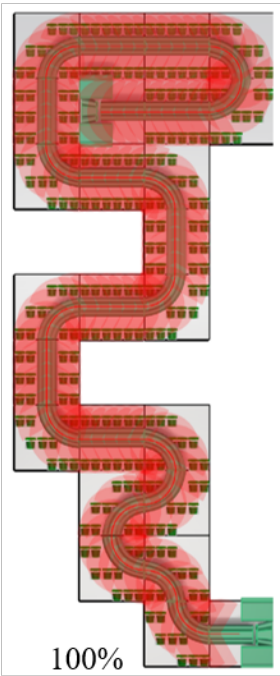

(e)

Figure 10. Robot reachability evaluation for vertical garden layouts that follow the proposed design guidelines. The track height is $10 \mathrm{~cm}$ for all the cases. The robot reachability of each case is overlaid in shaded color. The resultant percentage pot reachability is given under each case. $(\mathbf{a}-\mathbf{c})$ : closed track designs. (d,e): open track designs. It should be noted that the figures are not in the same scale.

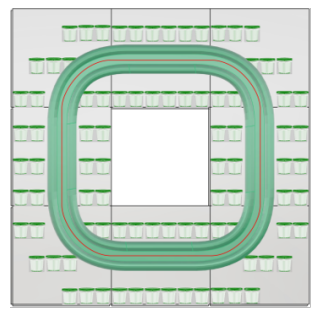

(a)

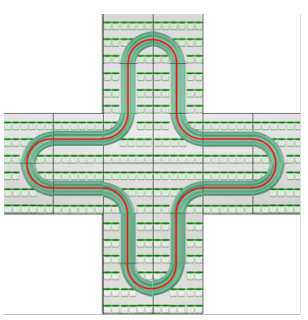

(d)

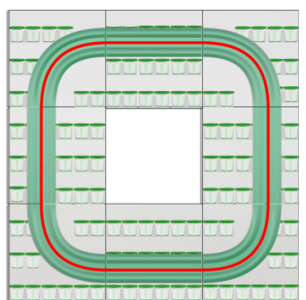

(b)

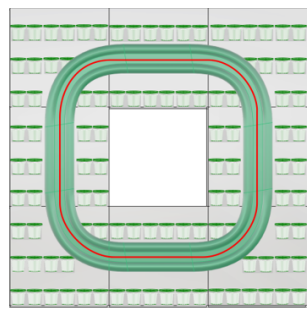

(c)

Figure 11. Vertical garden layouts created without attention to the introduced guidelines. The track height is $10 \mathrm{~cm}$ for all the cases other than (a). It should be noted that the figures are not in the same scale. (a): The track height is designed not according to the guidelines. Track height is $25 \mathrm{~cm}$. (b,c,e): The pot placing is not according to the guidelines. (d): An open track design.

A layout with only a different track height ( $25 \mathrm{~cm}$ in this case) from case 'a' of the layouts that followed the guidelines was used, as the test case ' $a$ ' of the layouts was not created considering the design guidelines (this case is depicted in Figure 12a). As mentioned under the guidelines for track height, a suitable track height specific to the robot should be utilised; otherwise, the robot reachability is adversely impacted. Due to 
the difference in the track height, many pots that the robot could previously reach with an appropriate track height now could not be reached. The number of reachable pots is reduced to $58.3 \%$ in this case. This result confirms the requirement of a rule for determining the track height as well as the ability of the proposed design.

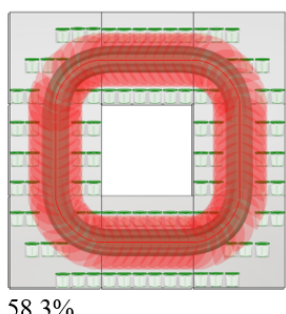

(a)

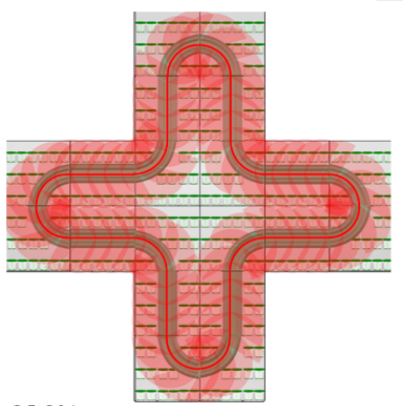

(d)

$85.2 \%$

$(\mathrm{d})$

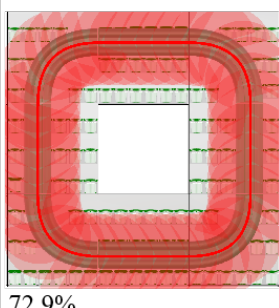

(b)

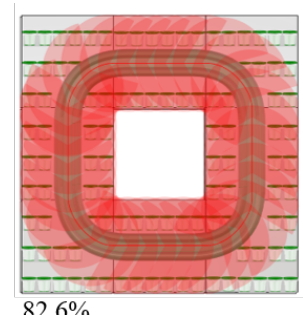

(c)
Figure 12. Robot reachability evaluation for vertical garden layouts that do not follows the proposed design guidelines. The robot reachability of each case is overlaid in shaded color. The resultant percentage pot reachability is given under each case. The track height is $10 \mathrm{~cm}$ for all the cases other than (a). It should be noted that the figures are not in the same scale. (a): The track height is designed not according to the guidelines. Track height is $25 \mathrm{~cm}$. (b,c,e): The plot placing is not according to the guidelines. (d): An open track design.

The control cases ' $b$ ', ' $c$ ', and ' $d$ ' consider plant pot layouts (given in Figure 12a-c, respectively), which the pots are not placed as per the guidelines. Since the pots are not arranged considering the introduced design guidelines, some of the pots may have been placed away from the track. As a result, a considerable amount of pots cannot be reached by the robot. The case ' $e$ ' given in Figure 12e considers a layout where pots are placed on the track end blocks similar to other blocks. According to the robot reachability results, the pots placed on the track ends are not reachable by the robot. This result validates the necessity of directed design of the track ends by considering the introduced design guidelines. The loss in reachability degrades the intended goal of robot-aided vertical garden maintenance.

Therefore, it is essential to follow design guidelines for placing pots to improve the productivity of the robot. Overall, the robot reachability evaluation results confirm that the introduced robot-inclusive design for vertical gardens can improve a robot's productivity. Furthermore, the cases that do not follow the design guidelines expose the necessity of following the intended robot-inclusive green wall design. Therefore, it can be concluded that the introduced robot-inclusive green wall design is beneficial for the development of robot-aided maintenance of vertical gardening.

\subsection{Analysis of the Proposed Design in Relation to Scalability and Customisability}

Design explorations of different layouts of modular tiles are created and analysed in relation to scalability and customisability. The iterations of the layout is limited to an area of $7.2 \mathrm{~m}$ by $7.2 \mathrm{~m}$, in a $12 \times 12$ grid, for a total of 144 tile placements. Seven layouts consisting of different compositions of tiles in various arrangements within this spanning area have been created in this regard. It should be noted that all the layouts considered 
here follow the proposed design guidelines for manipulability and safety. The layouts considered for the analysis along with reachability results are given in Figure 13. The effective green area covered on the wall through the overall layout of the vertical garden can be simplified by counting the overall number of pots reachable by the Urodela robot manipulator arm along the track. The number of reachable pots per unit length of the track, $\eta$ (defined in Equation (1)) is a crucial feature for track-based robot-aided maintenance of green walls. Therefore, the variation of $\eta$ for the test layouts were evaluated. The key parameters corresponding to the cases are given in Table 1.

$$
\eta=\frac{\text { Numberofreachablepots }}{\text { Totallengthofthetrack }}
$$

The layout of the case 'a' consisted of 20 horizontal-track tiles, 20 vertical-track tiles, 4 curved-track tiles and 2 end-track tiles on a test area of $7.2 \mathrm{~m}$ by $7.2 \mathrm{~m}$. This composition of tiles allowed the formation of the layout depicted in Figure 13a. The layout makes a green wall area of $16.56 \mathrm{~m}$ out of $51.84 \mathrm{~m}$, yielding to an effective green wall area of $31.9 \%$. The number of reachable pots was evaluated as 520, while the track length was $26.48 \mathrm{~m}$. Thus, the reachable pots per unit length of the track, $\eta$ was 19.63 pots $/ \mathrm{m}$.

(a)

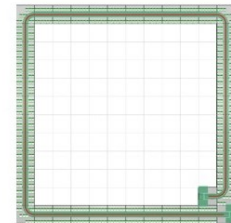

(b)

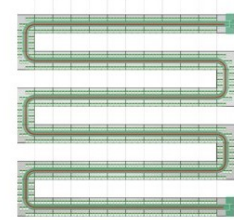

(c)

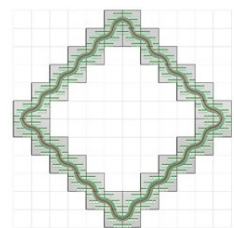

(d)

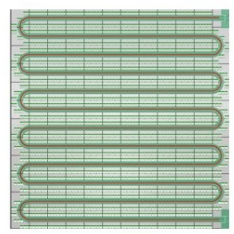

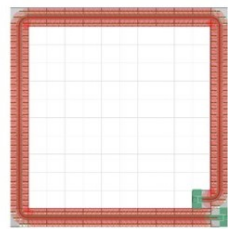
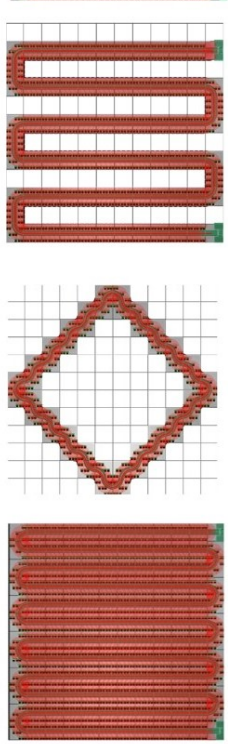

(e)

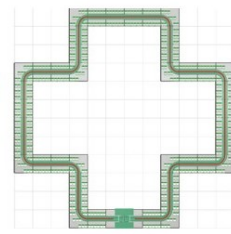

(f)

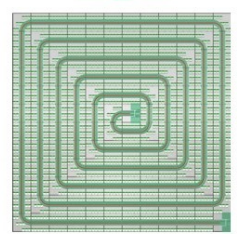

(g)

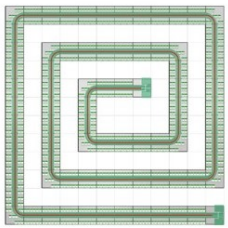

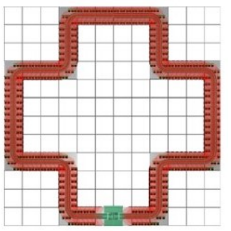
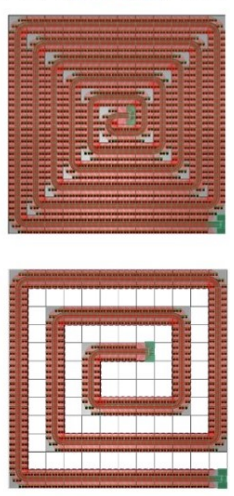

Figure 13. The test cases used for analysing the key features of the proposed design framework by using different test track layouts. The details of the test cases $(\mathbf{a}-\mathbf{g})$ are given in Table 1 . The robot reachability of each case is overlaid in shaded color. The reachability was $100 \%$ for all the cases.

Similarly, these parameters for each layout were evaluated. Based on the results of the analysis, the following salient features of the architectural design, which is useful for the scalability and customisability, could be identified.

- Layouts created using combinations of tiles could always achieve $100 \%$ robot reachability.

- Layouts with any composition of the proposed modules can make green walls such that the average number of reachable pots per unit length of the track is 20.1 pots $/ \mathrm{m}$. The variation of $\eta$ is minimal (standard deviation $=0.01$ ), even though different compositions were used. e.g., 'a': 19.63 pots $/ \mathrm{m}$, 'b': 19.86 pots $/ \mathrm{m}$, and ' $\mathrm{c}$ ': 21.22 pots $/ \mathrm{m}$. 
- Modular blocks can be arranged to form different amounts of effective green wall within a given spanning area/bounding box. e.g., 'a': $31.9 \%$, 'b': $53.5 \%$, and 'd': $100 \%$.

- Layouts can have different arrangements such as curves, horizontal and vertical tracks per users' requirement, even maintain the same level of effective green wall areas. e.g., ' $\mathrm{e}$ ' and ' $\mathrm{c}$ ' (straight and curve); ' $\mathrm{d}$ ' and ' $\mathrm{f}$ ' (horizontal and vertical).

Therefore, these features confirm the ability to scale and customise the proposed robot-inclusive design for green walls for different requirements such as arrangements, dimensions, and sizes, while maintaining an adequate level of performance for the robotaided maintenance of green walls.

Table 1. Results of the analysis of key features of the proposed design.

\begin{tabular}{|c|c|c|c|c|c|c|c|c|}
\hline \multirow{2}{*}{ Case } & \multicolumn{4}{|c|}{ Tile Composition } & \multirow{2}{*}{ Effective Area (\%) } & \multirow{2}{*}{ No. of Reachable Pots } & \multirow{2}{*}{ Track Length (m) } & \multirow{2}{*}{$\eta($ pots $/ \mathrm{m})$} \\
\hline & Horizontal & Vertical & Curved & Ends & & & & \\
\hline$a$ & 20 & 20 & 4 & 2 & 31.9 & 520 & 26.48 & 19.63 \\
\hline $\mathrm{b}$ & 60 & 5 & 10 & 2 & 53.5 & 880 & 44.31 & 19.86 \\
\hline c & 0 & 0 & 44 & 0 & 30.6 & 440 & 20.73 & 21.22 \\
\hline $\mathrm{d}$ & 120 & 0 & 22 & 2 & 100 & 1660 & 82.97 & 20.01 \\
\hline e & 14 & 16 & 12 & 2 & 30.6 & 480 & 24.25 & 19.79 \\
\hline $\mathrm{f}$ & 65 & 55 & 22 & 2 & 100 & 1684 & 84.17 & 20.01 \\
\hline $\mathrm{g}$ & 40 & 30 & 10 & 2 & 56.9 & 940 & 47.31 & 19.87 \\
\hline
\end{tabular}

\section{Conclusions}

Research has shown that robotics has been playing an increasingly important role to assist in maintenance and inspection tasks. As robotics technology progresses, it is essential to bridge the gap between roboticians and spatial designers in the design of both robots and its work environment. This paper seeks to fill the gap in vertical garden design for robot-inclusivity.

Design guidelines for robot-inclusive vertical gardens are developed based on a study of the limitations in maintenance robots operating in such environments. The study also includes a specific case study considering the Urodela robot [17], an existing vertical garden maintenance robot. These design guidelines can be sub-categorised into two design principles mainly arm manipulability and safety. Following this, an exemplary vertical garden setup is proposed as proof of concept for a robot-inclusive vertical garden. A modular approach to the vertical garden design was considered to provide ease of scalability and customisability for architects and designers.

Based on the proposed proof-of-concept design, the robot's productivity in terms of plant pot reachability has been evaluated on vertical gardens designed with the guidelines and other control cases. The simulations confirms the applicability of the proposed robot-inclusive green wall design by the productivity of a robot-aided vertical garden maintenance process. Overall, the implementation of this robot-inclusive vertical garden design is meant to complement maintenance tasks performed by manual labour. This implementation of robot-inclusive vertical garden infrastructure would help improve workplace safety and reduce costs incurred.

As the proposed design guidelines are derived mainly from the limitations of the Urodela robot, a further generalisation of guidelines would be beneficial for a similar class of wall-climbing robots to reduce the need for customisation. Future work could revisit the vertical garden design to enable track designs with junctions. Track switches on specialised track modules could be created to allow the robot to change its path during operation, to go on different branches of the track in future iterations. This would allow robot deployments on smaller-sized green vertical landscapes with multiple branching paths. Another direction to explore is the design of non-planar wall profiles for robot traversal. A study into the various wall profiles in architecture would allow designers to implement and generate different track layouts for robot traversal to suit the intended design. An 
artist's impression of the implemented modular robot-inclusive green wall landscape design is given in Figure 14 for an overall impression on how the project is implemented.

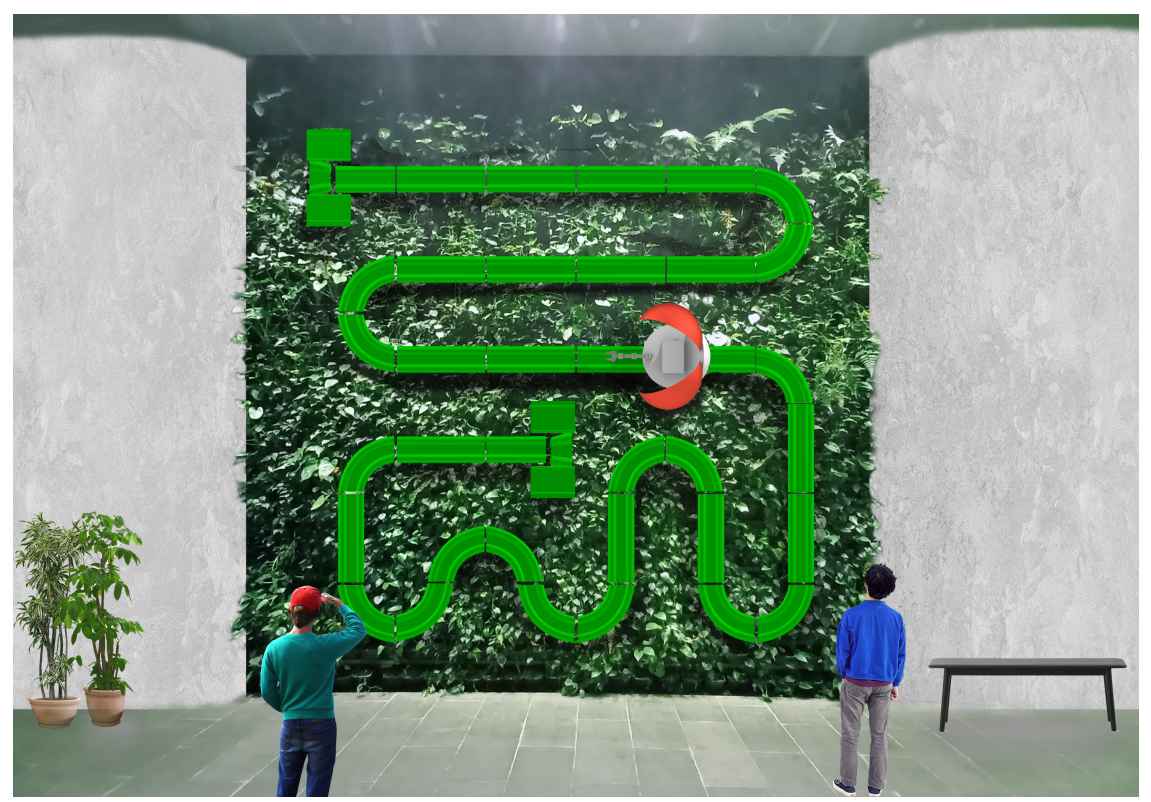

Figure 14. Artist's impression of implemented tiles.

Author Contributions: Conceptualization, M.S.K.Y. and M.R.E.; methodology, M.S.K.Y. and Q.B.N.; validation, S.M.B.P.S. and M.A.V.J.M.; formal analysis, S.M.B.P.S. and M.A.V.J.M.; investigation, M.S.K.Y. and M.A.V.J.M.; writing-original draft preparation, M.S.K.Y., S.M.B.P.S. and Q.B.N.; writing-review and editing, M.A.V.J.M., M.R.E.; visualization, M.S.K.Y.; supervision, M.R.E.; project administration, M.R.E.; funding acquisition, M.R.E. All authors have read and agreed to the published version of the manuscript.

Funding: This research is supported by the National Robotics Programme under its Robotics Enabling Capabilities and Technologies (Funding Agency Project No. 19225 00051), National Robotics Programme under its Robot Domain Specific (Funding Agency Project No. 19222 00058), National Robotics Programme under its Robotics Domain Specific (Funding Agency Project No. 19222 00108), and administered by the Agency for Science, Technology and Research.

Conflicts of Interest: The authors declare no conflict of interest. The funders had no role in the design of the study; in the collection, analyses, or interpretation of data; in the writing of the manuscript, or in the decision to publish the results.

\section{References}

1. Medl, A.; Stangl, R.; Florineth, F. Vertical greening systems-A review on recent technologies and research advancement. Build. Environ. 2017, 125, 227-239. [CrossRef]

2. Al-Kodmany, K. Sustainability and the 21st century vertical city: A review of design approaches of tall buildings. Buildings 2018, 8, 102. [CrossRef]

3. Radić, M.; Brković Dodig, M.; Auer, T. Green facades and living walls-A review establishing the classification of construction types and mapping the benefits. Sustainability 2019, 11, 4579. [CrossRef]

4. Rakhshandehroo, M.; Mohd Yusof, M.J.; Arabi, R. Living wall (vertical greening): Benefits and Threats. In Applied Mechanics and Materials; Trans Tech Publications Ltd.: Stafa, Switzerland, 2015; Volume 747, pp. 16-19. [CrossRef]

5. Wang, X.-X. From Nature to Cities: Patrick Blanc's Vertical Garden. Landsc. Archit. 2011, 5, 122-127. [CrossRef]

6. Madre, F.; Clergeau, P.; Machon, N.; Vergnes, A. Building biodiversity: Vegetated façades as habitats for spider and beetle assemblages. Glob. Ecol. Conserv. 2015, 3, 222-233. [CrossRef]

7. Min, J.; Kim, Y.; Lee, S.; Jang, T.W.; Kim, I.; Song, J. The fourth industrial revolution and its impact on occupational health and safety, worker's compensation and labor conditions. Saf. Health Work 2019, 10, 400-408. [CrossRef]

8. Muthugala, M.A.V.J.; Vega-Heredia, M.; Mohan, R.E.; Vishaal, S.R. Design and Control of a Wall Cleaning Robot with AdhesionAwareness. Symmetry 2020, 12, 122. [CrossRef]

9. Vega-Heredia, M.; Mohan, R.E.; Wen, T.Y.; Siti'Aisyah, J.; Vengadesh, A.; Ghanta, S.; Vinu, S. Design and modelling of a modular window cleaning robot. Autom. Constr. 2019, 103, 268-278. [CrossRef] 
10. Samarakoon, S.M.B.P.; Muthugala, M.A.V.J.; Le, A.V.; Elara, M.R. HTetro-infi: A reconfigurable floor cleaning robot with infinite morphologies. IEEE Access 2020, 8, 69816-69828. [CrossRef]

11. Quaglia, G.; Visconte, C.; Scimmi, L.S.; Melchiorre, M.; Cavallone, P.; Pastorelli, S. Robot arm and control architecture integration on a UGV for precision agriculture. In Proceedings of the IFToMM World Congress on Mechanism and Machine Science, Krakow, Poland, 15-18 July 2019; pp. 2339-2348.

12. Zhou, T.; Zhang, D.; Zhou, M.; Xi, H.; Chen, X. System Design of Tomatoes Harvesting Robot Based on Binocular Vision. In Proceedings of the 2018 Chinese Automation Congress (CAC), Xi'an, China, 30 November-2 December 2019; pp. 1114-1118.

13. Lauguico, S.C.; Concepcion, R.S.; Macasaet, D.D.; Alejandrino, J.D.; Bandala, A.A.; Dadios, E.P. Implementation of Inverse Kinematics for Crop-Harvesting Robotic Arm in Vertical Farming. In Proceedings of the 2019 IEEE International Conference on Cybernetics and Intelligent Systems (CIS) and IEEE Conference on Robotics, Automation and Mechatronics (RAM), Bangkok, Thailand, 18-20 November 2019; pp. 298-303.

14. Kim, H.G.; Park, D.H.; Chowdhury, O.R.; Shin, C.S.; Cho, Y.Y.; Park, J.W. Location-based intelligent robot management service model using RGPSi with AoA for vertical farm. In Advances in Computer Science and Its Applications; Springer: Berlin/Heidelberg, Germany, 2014; pp. 309-314.

15. Kim, J.; Kim, S.; Ju, C.; Son, H.I. Unmanned aerial vehicles in agriculture: A review of perspective of platform, control, and applications. IEEE Access 2019, 7, 105100-105115. [CrossRef]

16. Wilkinson, S. Wallbot: A must for green wall. In Built Environment Economist: Australia and New Zealand; Australian Institute of Quantity Surveyors: Sydney, Australia, 2020; p. 46.

17. Hayat, A.A.; Eduardo, S.C.; Muthugala, M.A.V.J.; Pinxian, A.L.; Elara, M.R. Urodela: Design of a Reconfigurable Robotic System for Landscapes Maintenance. In Proceedings of the 5th IEEE/IFToMM International Conference on Reconfigurable Mechanisms and Robots (ReMAR 2021), Toronto, ON, Canada, 12-14 August 2021. under review.

18. Bao, G.; Yao, P.; Cai, S.; Ying, S.; Yang, Q. Flexible pneumatic end-effector for agricultural robot: Design \& experiment. In Proceedings of the 2015 IEEE International Conference on Robotics and Biomimetics (ROBIO), Zhuhai, China, 6-9 December 2015; pp. 2175-2180.

19. Vidoni, R.; Gallo, R.; Ristorto, G.; Carabin, G.; Mazzetto, F.; Scalera, L.; Gasparetto, A. ByeLab: An agricultural mobile robot prototype for proximal sensing and precision farming. In Proceedings of the ASME International Mechanical Engineering Congress and Exposition. American Society of Mechanical Engineers, Tampa, FL, USA, 3-9 November 2017; Volume 58370, p. V04AT05A057.

20. Khan, A.; Noreen, I.; Ryu, H.; Doh, N.L.; Habib, Z. Online complete coverage path planning using two-way proximity search. Intell. Serv. Robot. 2017, 10, 229-240. [CrossRef]

21. Anshel, J. Visual ergonomics in the workplace. In Proceedings of the ASSE Professional Development Conference and Exhibition. American Society of Safety Engineers, Baltimore, MD, USA, 13-16 June 1999.

22. Fernandez, J.E. Ergonomics in the workplace. Facilities 1995, 13, 20-27. [CrossRef]

23. Elara, M.R.; Rojas, N.; Chua, A. Design principles for robot inclusive spaces: A case study with roomba. In Proceedings of the 2014 IEEE International Conference on Robotics and Automation (ICRA), Hong Kong, China, 31 May-7 June 2014; pp. 5593-5599.

24. Sosa, R.; Montiel, M.; Sandoval, E.B.; Mohan, R.E. Robot ergonomics: Towards human-centred and robot-inclusive design. In Proceedings of the DS 92: Proceedings of the DESIGN 2018 15th International Design Conference, Dubrovnik, Croatia, 21-24 May 2018; pp. 2323-2334.

25. Tan, N.; Mohan, R.E.; Watanabe, A. Toward a framework for robot-inclusive environments. Autom. Constr. 2016, 69, 68-78. [CrossRef]

26. Manso, M.; Castro-Gomes, J. Green wall systems: A review of their characteristics. Renew. Sustain. Energy Rev. 2015, 41, 863-871. [CrossRef]

27. Pérez-Urrestarazu, L.; Urrestarazu, M. Vertical greening systems: Irrigation and maintenance. In Nature Based Strategies for Urban and Building Sustainability; Elsevier: Amsterdam, The Netherlands, 2018; pp. 55-63.

28. Giacomello, E. Case study: Bosco verticale, Milan: A new urban forest rises in Milan. CTBUH J. 2015, 1, $12-18$.

29. Vertical Forest. 2020. Available online: https://www.stefanoboeriarchitetti.net/en/project/vertical-forest/ (accessed on 22 April 2021).

30. Ibrahim Momtaz, R. Vertical garden as a sustainble urban prespective in Cairo. JES J. Eng. Sci. 2018, 46, 246-262. [CrossRef]

31. Arad, B.; Balendonck, J.; Barth, R.; Ben-Shahar, O.; Edan, Y.; Hellström, T.; Hemming, J.; Kurtser, P.; Ringdahl, O.; Tielen, T.; et al. Development of a sweet pepper harvesting robot. J. Field Robot. 2020, 37, 1027-1039. [CrossRef]

32. Samarakoon, S.M.B.P.; Muthugala, M.A.V.J.; Elara, M.R.; Kumaran, S. Toward Pleomorphic Reconfigurable Robots for Optimum Coverage. Complexity 2021, 2021, 3705365. [CrossRef]

33. Muthugala, M.A.V.J.; Vengadesh, A.; Wu, X.; Elara, M.R.; Iwase, M.; Sun, L.; Hao, J. Expressing attention requirement of a floor cleaning robot through interactive lights. Autom. Constr. 2020, 110, 103015. [CrossRef]

34. Zhang, B.; Xie, Y.; Zhou, J.; Wang, K.; Zhang, Z. State-of-the-art robotic grippers, grasping and control strategies, as well as their applications in agricultural robots: A review. Comput. Electron. Agric. 2020, 177, 105694. [CrossRef]

35. Muthugala, M.A.V.J.; Samarakoon, S.M.B.P.; Elara, M.R. Tradeoff between area coverage and energy usage of a self-reconfigurable floor cleaning robot based on user preference. IEEE Access 2020, 8, 76267-76275. [CrossRef] 
36. Rose, D.H.; Meyer, A. A Practical Reader in Universal Design for Learning; Harvard Education Press: Cambridge, MA, USA, 2006.

37. Steinfeld, E.; Maisel, J. Universal Design: Creating Inclusive Environments; John Wiley \& Sons: Hoboken, NJ, USA, 2012. 